\title{
DYNAMIC QUALITY OF SERVICE STABILITY BASED MULTICAST ROUTING PROTOCOL FOR MANETS (DQSMRP)
}

\author{
M.Vijayalakshmi ${ }^{1}$, and Dr.D.SreenivasaRao ${ }^{2}$ \\ ${ }^{1}$ Department of Electronics and Communication Engineering, GNITS, India \\ mvlakshmi_gnits@yahoo.co.in \\ ${ }^{2}$ Department of Electronics and Communication Engineering, JNTUH, India \\ dsraoece@gmail.com
}

\begin{abstract}
Mobile ad hoc networking does not possess any fixed infrastructure and hence, stable routing is the major problem. The mobility nature of MANET's node facilitates rediscovery of a new path to organizing a routing. In order to intensify the Quality of Service and routing stability in MANET, we propose a Dynamic Quality of service Stability based Multicast Routing Protocol by modifying the Cuckoo Search Algorithm through a modernizing mechanism which is derived from the differential evolution algorithm. Tuned CSA is a combined feature of CSA and DE algorithms. Periodically, each node in the network creates neighbour stability and QoS database at every node by calculating the parameters like node and link stability factor, bandwidth availability, and delays. Finally, multicast path constructs route request and route reply packets, stability information and performing route maintenance.
\end{abstract}

\section{KEYWORDS}

Manets, QoS, Tuned CSA, DQSMRP

\section{INTRODUCTION}

Mobile Ad hoc Networks (MANETs) are foremost in class kind of wireless communication networks in which mobile nodes link on an on-demand basis. MANETs are having the ability to configure on their own and easing peer-level communications among mobile nodes independent by having no fixed infrastructure. When the route traffic occurs, constructing a MANET with each node's to continuously retain the information remains a major challenge. Normally, MANETs are used for group communications where multicast protocols are efficiently compared to unicast protocols since they improve the efficiency of the wireless links in MANETs. The multicast protocols are also used as application demands for transmitting copies from either single or multiple sources to the receivers.

Multicasting reduces the communication costs by sending the single copy of the data to multiple recipients through which it minimizes link bandwidth, processing and transmission delay. Previous researchers have developed different algorithms to improvise the ability for selecting the

David C. Wyld et al. (Eds) : CSITA, ISPR, ARIN, DMAP, CCSIT, AISC, SIPP, PDCTA, SOEN - 2017

pp. 159- 173, 2017. (C) CS \& IT-CSCP 2017

DOI : $10.5121 /$ csit.2017.70116 
routing path(s) and thereby, effectively met the desired Quality of Service (QoS) [1]. This infrastructure results in a highly dynamic topology causing a challenging task for QoS Routing .

When compared with well-established networks such as Wi-Fi, Global System for Mobile communication (GSM) and Code Division Multiple Access (CDMA), MANET was not reliable to meet QoS performances. The MANET performance is determined by the QoS through the basic parameters like bandwidth or delay or loss. QoS routing addresses both the issues of deciding a path from source to destination and fulfilling the QoS constraints like bandwidth, delay and loss. Route selection method is used to fulfill QoS requirements. The goal of QoS routing strategy is to maximize the network resources in order to reduce the constraints in MANET's mobile nodes.

In real time applications, QoS routing protocol works based on multi-hop mobile networks [3, 4]. Particularly, QoS requirement depends on constraints such as link constraints, path constraints and tree constraints. End to End delay is usually affected by path constraint whereas, delay-jitter [5] by tree constraint. In order to meet all the constraints, robust techniques are required to solve the QoS requirements. Heuristic techniques are better to solve these constraints rather than deterministic methods. Researchers have studied various improvements based on heuristic methods $[5,6]$ and at the same time devised various solutions to solve QoS routing problems using heuristic methods [7].

Ad hoc mobile routing (AM Routing) was studied through which a specific unicast routing protocol was identified as more feasible than other unicast routing protocols (8). In QoS routing, network topology was constructed in which bandwidth link works in destination point [9]. A new protocol was proposed for ad hoc networks which are used to establish and maintain a shared mesh for each multicast group called Policy-based Unified Multi-radio, Architecture for Agile Mesh Networking (PUMA) [10]. A new single mixed metrics have been delineated for multiconstraint QoS routing [11]. Previous literaturehas found that Agent Based Multicast Routing Scheme (ABMRS) on MANET's could be used as a set of static and mobile agents for QoS routing $[12,13]$. Optimized link state routing protocol was launched and scrutinized in different research papers [14].

Besides that, several heuristic methods are examined to study the multi-constraint QoS routing. In [15], Simulated Annealing (SA) was introduced to solve the QoS routing problems. Reference [16] discussed Bees Life Algorithm (BLA) to solve the QoS multicast routing problem and constraints such as delay, allowed jitter, and requested bandwidth have been studied. Similarly, a Tabu search [17] based method concentrates on two important constraints in QoS such as bandwidth and end-to-end delay. Ant algorithm optimization was globally accepted for the effective and systematic handling of QoS routing [18, 19]. Harmony search (HS) algorithm, a unique algorithm was used to find the path of the MANET and it was discussed based on bandwidth-delay-constraints in multicast routing problem [20].

Hybrid methods have been proposed to address the delay of premature convergence [21]. A PSOGA algorithm was evolved using a combination of both particle swarm optimization (PSO) method and genetic algorithm (GA) which provides an effective and efficient search for the solution. In [22], the new fuzzy genetic algorithm is also introduced for QoS multicast routing is also discussed. 
Thus in this paper, we propose Dynamic Quality of Service Stability based multicast routing protocol (DQSMRP) for the multi-constraint QoS routing problem. Recently, Cuckoo Search (CS) is derived from reproduction strategies [23] which are widely used in heuristic based algorithms. [24, 25].It is more exhaustive and provides quick search to obtain better solutions to routing in more reliable and robust manner.

\section{PROBlem Definition, ReSEARCH Methodology}

The optimization problem is used to solve the QoS routing problem which is the primary objective to find a multicast tree constraints based on the cost function in order to reduce the practical constraints. Four different constraints are considered for formulating the current study such as delay, packet loss, bandwidth and jitter. The following equation provides formulation data for computation [5] QoS routing function is formulated as:

$$
\operatorname{MinC}(H(x, s))=C_{c}+\delta_{1} C_{b}+\delta_{2} C_{d}+\delta_{3} C_{d j}+\delta_{4} C_{p l}
$$

Reasons to merge both cost optimization and multi-constrained routing are as follows:

$>$ Formulations are based on multiple constraints and the multicast structure in order to spot an attainable path for each node having source and destination.

$>$ To shrink the network resource utilization by users.

In progressively,

$$
C(H(x, s))=\sum_{e \in H(x, S)} c(y)
$$

The Delay Constraint is defined as the allowed delay limit that exists in any branch of the network. When there is no link provided between any two nodes, a penalty occurs.

$$
\operatorname{Delay}(R(x, y))=\sum_{\theta \in R(x, y)} d l(x)+\sum_{n \in R(x, y)} d l(y)
$$

The Bandwidth Constraint is the requirement of average bandwidth among all tree branches in the network.

$\operatorname{Bandwidth}(R(x, y))=\min (b w(x)), x \in R(x, y)$

Specifically $\delta_{1}, \delta_{2}, \delta_{3}, \delta_{4}$, are the bandwidth, delay, delay-jitter and packet-loss for the corresponding penalty constants. The objective of the initiated solution is to solve the multicast QoS routing problem as described by numerical experiments in the coming section. 


\subsection{Tuned Cuckoo Search Multicast Routing Protocol}

A technique that produces simple implementation and quality solutions is the best solution provider. This paper provides better results over the existing algorithms such as meta-heuristic techniques. Before analyzing the proposed method, the cuckoo algorithm is discussed as follows.

\subsubsection{An Overview of Cuckoo Search Algorithm}

By analogy of reproduction strategy, CS algorithm is a well known prominent heuristic search algorithms which follow the principle of cuckoos (chosen nests of other birds for laying eggs) $[26,27]$. Generally, the host bird would not permit the cuckoo eggs to easily differentiate and vice-versa. The important optimization procedure is as follows:

- Initial Solution: Cuckoo egg symbolizes a set of solutions and its value of dimensions was extracted from various nests randomly;

- Next Generation: The best eggs with common solutions only able to proceed to the next generation.

- Acceptance Rule: New egg will succeed in the nest if eggs are unknown

Important step of CS are explained as follows:

- Step 1-Randomly set“Pop” host nests, a group of the population. (e.g., $P=\sum_{i=1}^{p o p} P_{i}$ )

- Step 2-Evaluate the fitness of all nests $F=\sum_{i=1}^{p o p} f\left(P_{i}\right)$

- $\quad$ Step 3- Do Iterations < Max.Iterations

- Step 4- Produce a cuckoo egg $P^{i}$ by applying a Lévy flight from a random nest

- Step 5-Compute the fitness of $f\left(P_{i}\right)$

- Step 6-Check for existence: choose again a random nest $J$

- Step 7-if $f\left(p_{i}^{1}\right)<f\left(P_{i}\right)$ then

- $\quad$ Step 8- $P_{j=} p_{i}^{1}$

- Step 9-end if

- Step 10-Remove a fraction of the worst nests at the rate of $R_{c}^{\square}$

- Step 11- Randomly, Building new nests at new locations using Levy flights replacements

- Step 12-End While 
The benefit of this heuristics is its simplicity. The single parameter of CS algorithm should be considered enough when compared with PSO and Ant colony algorithms.

\subsubsection{Node Stability}

For efficient better packet delivery services in forwarding group, the mobile node is to be very stable .The way of providing stability is that moving node around its current position. Node stability metric have been used in previous work of authors is given in [28]. It is studied that stable path nodes have been found from packets from the source to the multicast group. Selfstability and neighbor nodes stability are two metrics to represent node stability.

Following steps to find stability of a node:

$>$ In MANET's nodes, it establishes self-stability, i.e., node movement is relative to its previous position.

$>$ MANET's node gives the node stability factor from self-stability and neighbor stability. Actually, stability is based on the distance between the movements of nodes positioning within transmission range. Stable node is created when movement within transmission range.

\subsubsection{Neighbor node stability}

It can be defined as how the node is being connected to its neighbour to obtain self-stability. Whenever nodes are in transmission range, it can exchange messages with each other. Each node consists of connectivity information, the signal stability of one-hopneighbors, and also maintains a neighbor list. The degree of a node $\mathrm{n}$ is denoted as number of links (or nodes) which connected to it and denoted as ND.

$N_{s}(t)=\propto \times \frac{1}{N D} \sum_{i=1}^{N D} S_{s}^{i}(t)+(1-\propto) \times N_{s}(t-1)$

Where, $\alpha$ is the weighting factor (lies between 0 and 1) and is distributed between 0.6 and 0.7 since they yield better results in simulation.

\subsubsection{Link Stability}

Link stability between the nodes defines quality and existence of the connection. It is reckoned based on two parameters:

- Received Signal Strength and

- A lifetime of the Link.

- The algorithm 1 represents a pseudocode for updating link stability status between the nodes. The different parameters used in the algorithm are as follows: 
- Lifetime - Duration of continuous connectivity between the nodes

- Lifetime threshold - Indicates the maximum limit of link lifetime that decides link stability

- Link stability status - It is a Boolean variable that defines link stability between the nodes.

- Recent - Indicates most recent response received for a Hello packet from a neighbor

- $\mathrm{P}$ - Represents Total Number of Hello packets

- Received signal strength - Represents the strength of the signal received from a neighbor

- Signal threshold - Is acceptable signal strength to be received from neighbors

\subsubsection{Algorithm: Link stability status between the nodes}

- Step 1: P = No of Hello Packets;

- $\quad$ Step 2: Lifetime =0;

- Step 3: Link stability status $=0$;

- $\quad$ Step 4: Recent =0;

- Step 5: Lifetime threshold $=\mathrm{P} \times$ Hello Packet Interval;

- Step 6: While P >0 do

- Step 7: If received signal strength $\geq$ signal threshold then

- Step 8: Lifetime = lifetime + 1;

- $\quad$ Step 9: Recent = 1;

- Step 10: $\mathrm{P}=\mathrm{P}-1$;

- Step 11: else

- $\quad$ Step 12: Recent =0;

- Step 13: $\mathrm{P}=\mathrm{P}-1$;

- Step 14: end if

- Step 15: end while

- Step 16: lifetime sec $=$ lifetime $\times$ Hello Packet Interval;

- Step 17: if (lifetime sec > lifetime threshold) and (Recent)

- Then

- Step 18: link stability status $=1$;

- Step 19: else

- $\quad$ Step 20: link stability status $=0$; 
- Step 21: end if

\subsubsection{Delay Estimation}

For delay estimation, an arbitrary node that contributes to traffic forwarding using the M/M/1 queuing system is modeled. This queue represents a single queuing station with a single server [29]. The authors assume that the contributing nodes are served by a single server with first come first serve queuing policy. Packets arrive according to a Poisson distribution rate $(\lambda)$, and the probability distribution of the service rate is exponential, denoted by $\mu$. The maximum size of the queue in every node is represented by $\mathrm{K}$.

To satisfy delay requirements in multimedia real-time applications, packets must be received by multicast receivers which satisfy the application delay constraints. When a packet is to be sent either by a source node or forwarding group of nodes, it experiences three types of delays: queuing, contention and transmission delay. The total delay considered over a link between two nodes is given by

$$
d_{\text {Total }}=d_{q}+d_{c}+d_{t}
$$

The queuing delay denoted by $d_{q}$ is the delay between the time the packet is assigned to a queue and the time it starts transmission. The packet waits and gives other packets in transmission queue are transmitted at this particular time. This is the amount of time a packet is spent in the interfacing queue $(\mathrm{T})$.

\subsubsection{Bandwidth Estimation}

Bandwidth is one of the key principles for assessing Quality of service (QoS). The authors considered their previous work presented in [30], to calculate the available bandwidth based on their channel status of the radio link to calculate the idle and busy periods of the shared wireless media. Bandwidth is measured by the node activities and its surrounding neighbors using the channel utility.

In IEEE 802.11 MANETs, once it is achieved to gain the channel access, a node can be able to transmitting data packets. Hence, a node senses the channel and estimates bandwidth by using the idle and busy times in a predefined interval. This is expressed by the following equation.

$$
B W=\frac{T_{\text {idle }}}{T_{\text {interval }}} \times C
$$

\subsubsection{Route Discovery Process using Tuned Cuckoo Process}

Multicast stable QoS path creation involves two phases: a request and a reply phase. Request phase invokes route discovery process to find routes having a group of receivers using stable and QoS intermediate nodes. It helps to reply phase for updating of RIC and also performs confirmation process. It acts as intermediate nodes that help to create a multicast mesh to the group of receivers.

RR packets are used in source node to find the route to its group of receivers. The sequences of operations that occur are as follows: 
- Source node prepares an RR packet with an application of bandwidth and delay requirements.

- Selective transmission of RR packet to neighbors once satisfies stability criteria, i.e., SFBN greater than SFTH, and bandwidth requirement, i.e., estimated bandwidth greater than twice the application requirements.

- Once received, node discards RR packet (both sequence number and source address).

- If RR packet is not a duplicate, check if the availability of Routing Information Cache (RIC); if available, RP packet create and start reply propagation to the source.

- If RR packet is a duplicate, then discard it and stop transmission of RR packet.

- If not duplicate and no route available in RIC, transmit the RR packet by updating its fields (route record, Stability Factor Between Nodes (SFBN ) record, bandwidth record, delay record, time to live, and nexthop address) to its neighbors as in step 2.

- Perform steps 3 to 6 (Till destination reached).

- If the receiver is not reached within certain hops, send RE packet to the source node.

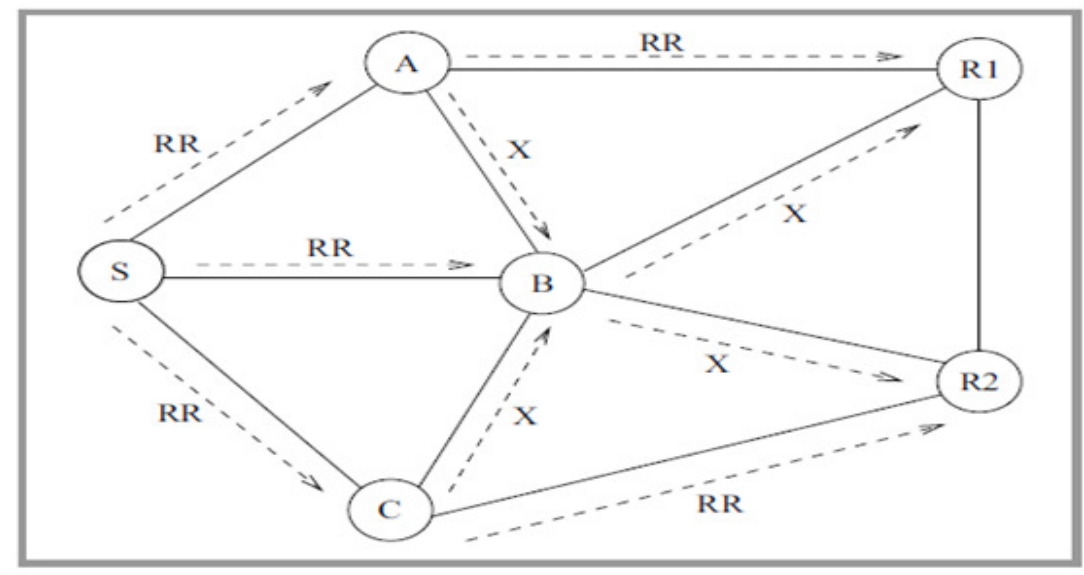

Figure 1: Route request paths from S to R1 and R2

\section{RESULTS AND DISCUSSION}

\subsection{Simulations and Performance Evaluation}

In this section, the performance of proposed protocol with DQSMRP and Fully Distributed Multicast Routing Protocol (FDMRP) [31] is compared, through a considerable set of simulations. These etiquettes have been taken for comparison because both are mesh based. These protocols are collated in terms of packet delivery ratio, control overhead and average end-to-end delay. Simulations considering the values of the production parameters are taken for several iterations, and these are computing the mean. The values lying within $95 \%$ of the confidence 
interval of the mean are used for computing the mean value, which is shown in the graphs in result analysis section. The various network scenarios have been simulated. Simulation environment consists of four models: Network, Channel, Mobility and Traffic. An ad hoc network is created at an area of $1 \times \mathrm{b}$ square meters as network model; it is having $\mathrm{N}$ number of mobile nodes spaced randomly. Limited bandwidths in coverage area around each node have shared to its neighbors. It is assumed that the operating range of transmitted power and communication range are constant.

\subsubsection{Performance Parameters}

Following metrics have been used to scrutinize the performance:

- Packet Delivery Ratio (PDR) : The ratio of a number of average data packets received at the multicast receivers to the number of data packets sent by the source.

- Packet Over head : The ratio of control packets sent to the network to the total number of average data packets delivered to the receivers.

- Average end-to-end Delay: The average delay experienced by the successfully delivered packets in reaching their receiver.

Table 1: Simulation scenario

\begin{tabular}{|l|l|}
\hline No. of Nodes & $50,100,150$ and 200. \\
\hline Routing Protocol & DQSMRP \\
\hline Area Size & 1000 X 1000 \\
\hline Mac & 802.11 \\
\hline Radio Range & $250 \mathrm{~m}$ \\
\hline Simulation Time & $10 \mathrm{sec}$ \\
\hline Traffic Source & CBR \\
\hline Packet Size & 512 \\
\hline Receiving Power & 0.395 \\
\hline Sending power & 0.660 \\
\hline Idle Power & 0.035 \\
\hline Initial Energy & $10.0 \mathrm{~J}$ \\
\hline Node Speed & $5,10,10$ and,20 ms \\
\hline No of destinations & $2,4,6$ and 8 \\
\hline
\end{tabular}




\subsection{Result Analysis}

The simulation results of DQSMRP and FDMRP present the comparison results by varying number of multiple destinations and node speed. According to the simulation results in Fig 2 to Fig 7, the performance of TCSMRP is more reliable than FDMRP. The execution of DQSMRP packet delivery ratio is gathered more than $14.2 \%$ compare to FDMRP. Moreover, the rendering of DQSMRP startsto end delay is less than FDMRP. The performance rate is almost $18 \%$ less and the delay ratio is varied with respective of node speed and number of destinations. According to the overhead performance scenario, the overhead rate is $22 \%$ less than FDMRP.

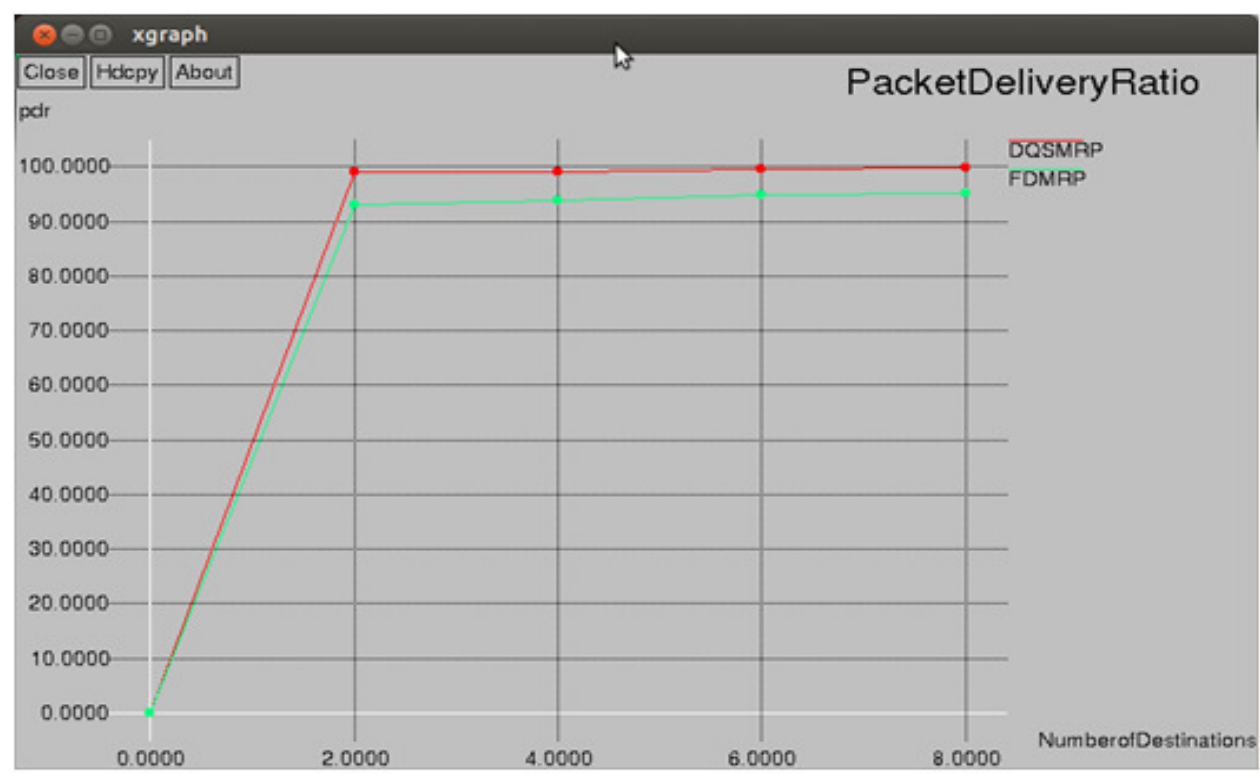

Figure 2: PDR vs Number of Destinations

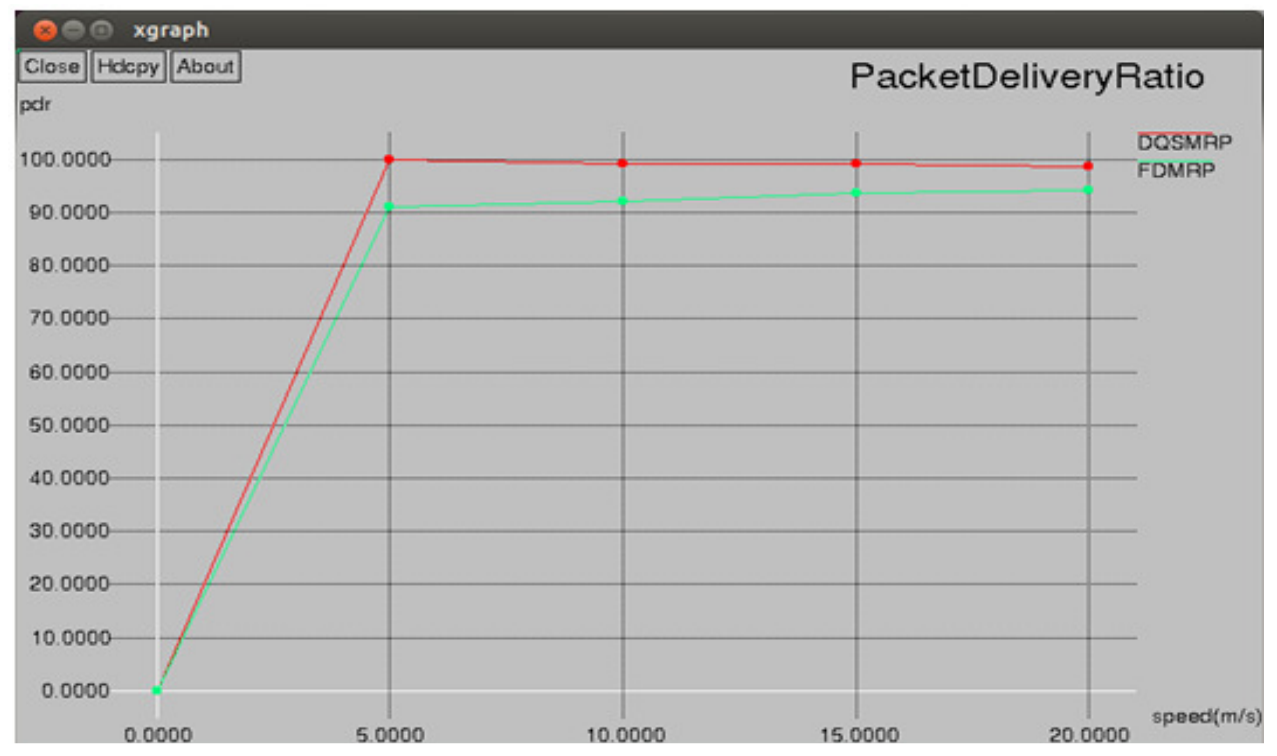

Figure 3: PDR vs Node Speed 


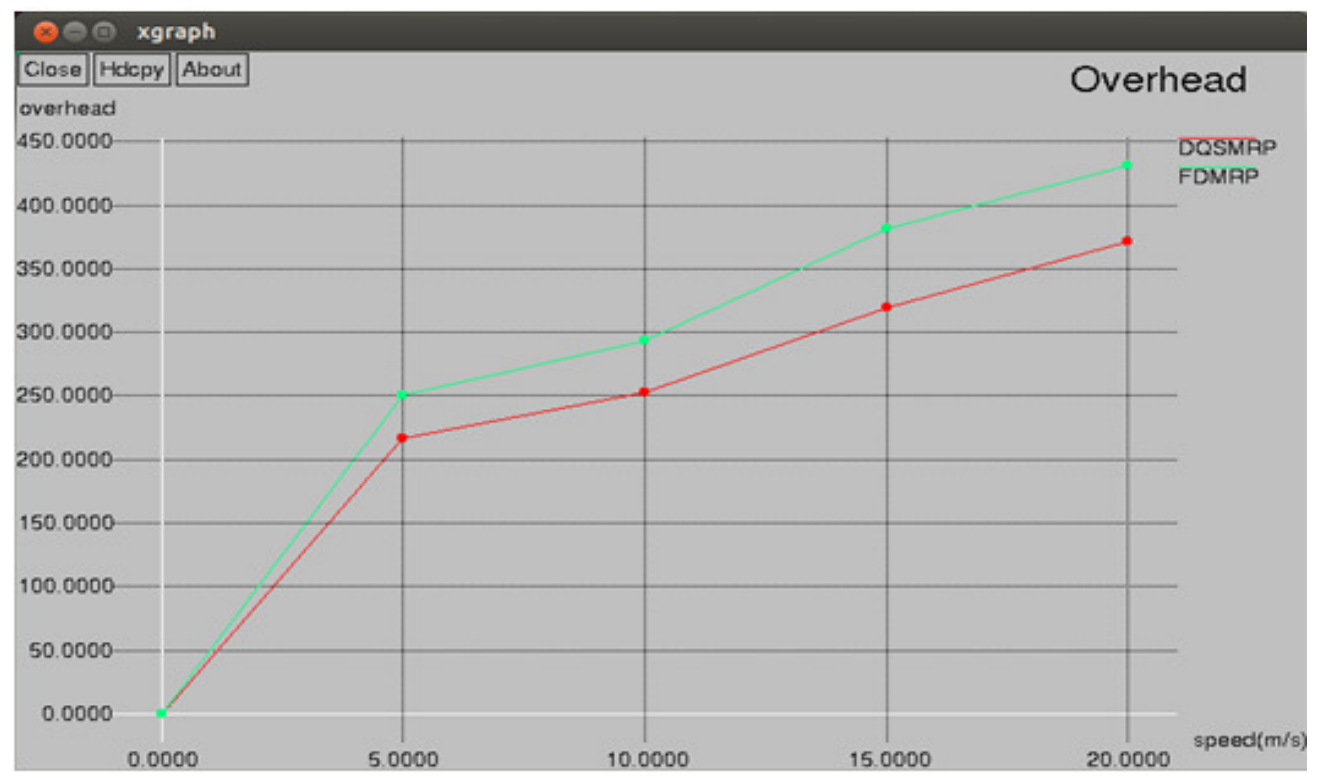

Figure 4: Overhead vs Node Speed

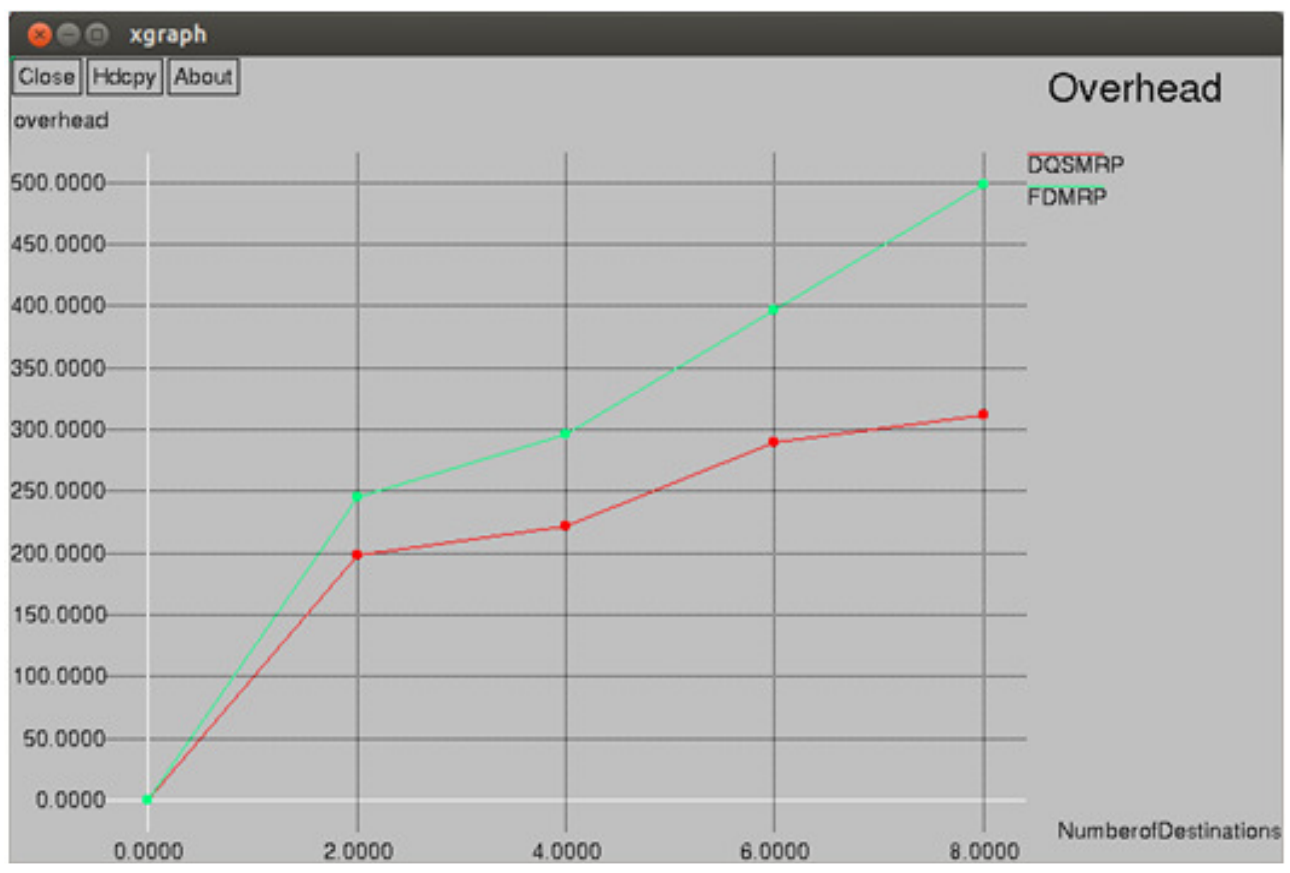

Figure 5: Overhead vs Number of destinations 


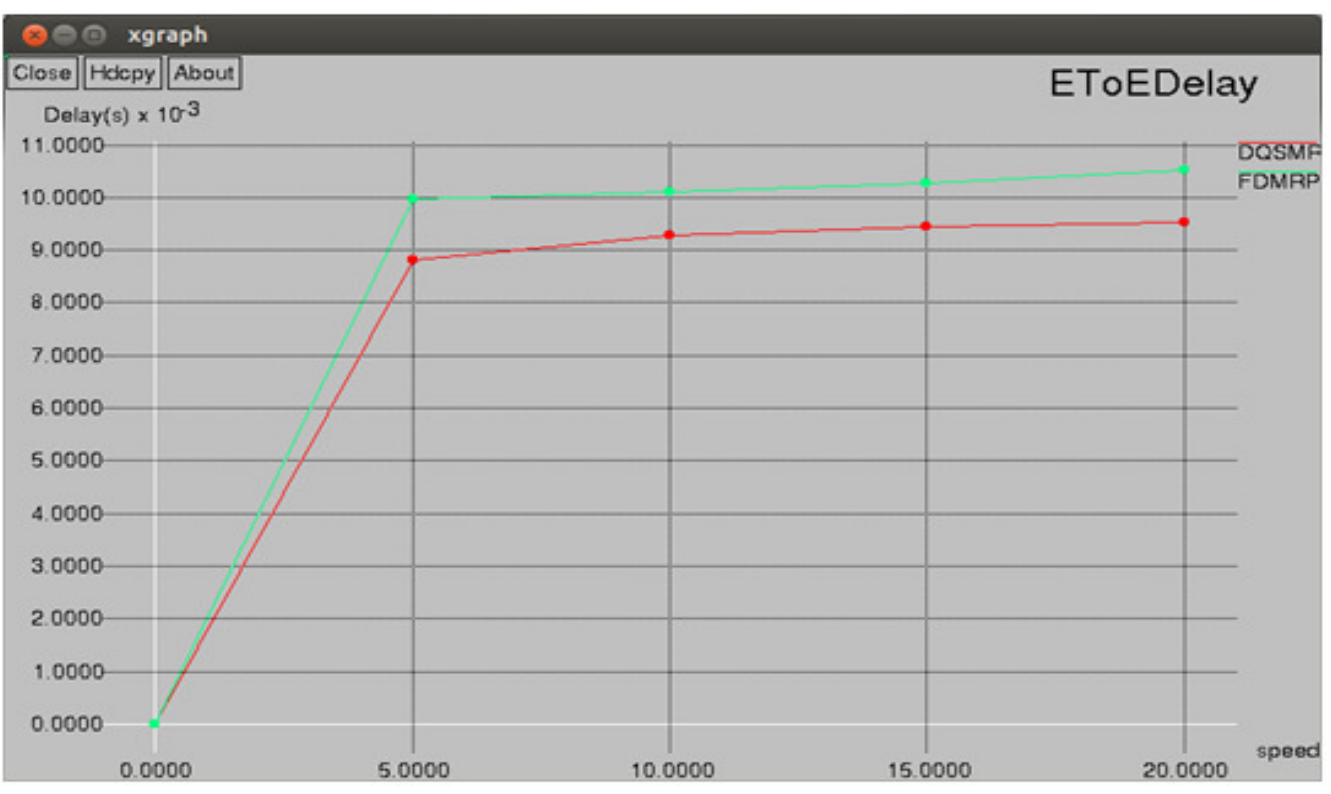

Figure 6: End - End Delays vs Speed

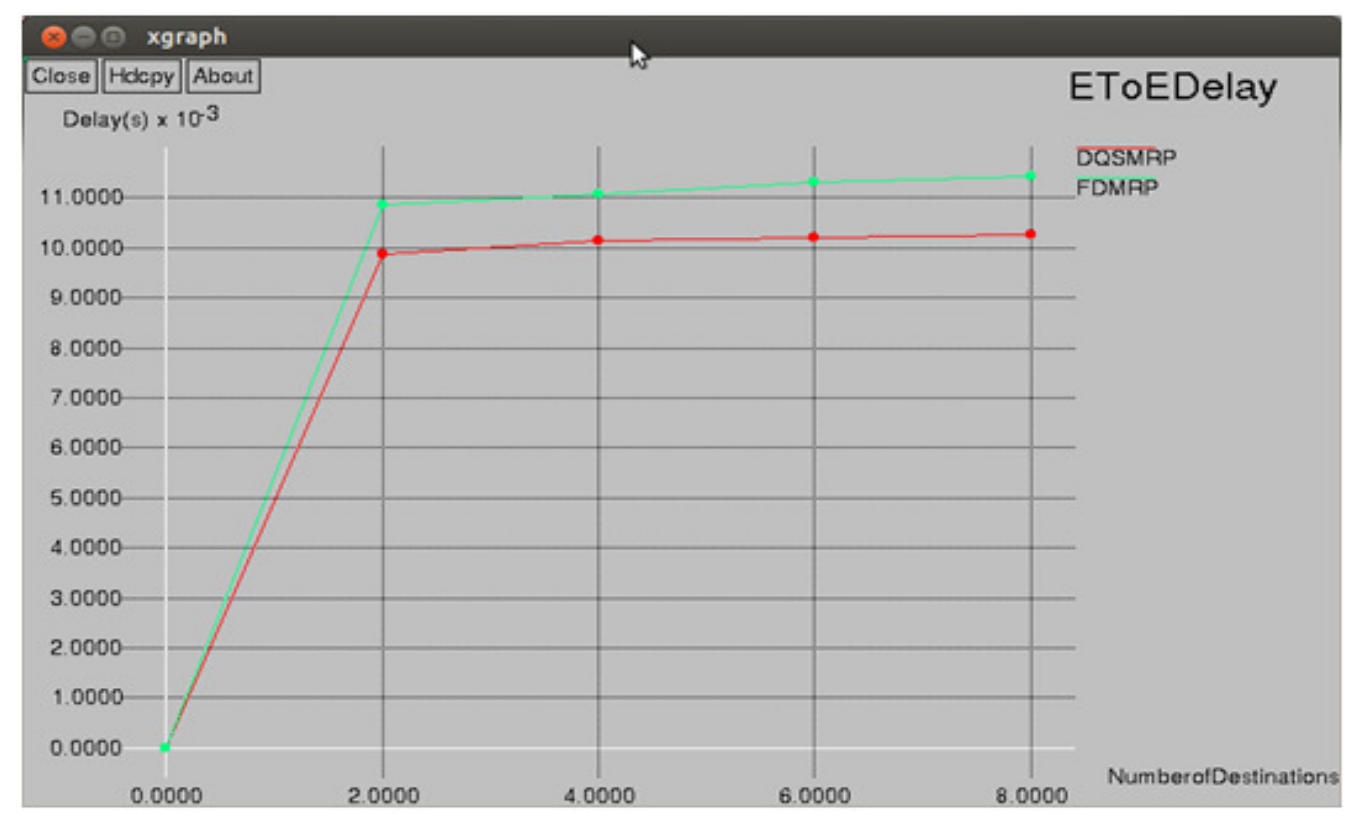

Figure 7: End - End Delays vs Number of Destinations

\section{CONCLUSiOnS}

This paper clarifies about a hybrid computational intelligent algorithm i.e., Tuned Cuckoo Search Algorithm (TCSA), that includes the dominant attribute of two different heuristic techniques have been used to solve QoS multicast routing. The QoS routing in a MANET is a complicated to determine a prime routing. The general conclusion from presented simulation experiments reveals that proposed routing protocol performs better than FDMRP in terms of packet delivery ratio, 
packet overhead and average delay from start to end as a function of varying number of receivers, sources and node speeds. In future works, the author's aims to study further by comparing our Tuned Cuckoo Search Multicast Routing Protocol with some more QoS based routing protocols in MANETs. Numerical experiments have shown that the propounded hybrid TCSA algorithm provides superior search multicast trees, particularly in both swift convergence and robustness, when compared to other algorithms. This proposed algorithm shall be the lead for real-time networks with suitable refinement based on when and where it utilized.

\section{REFERENCES}

[1] R. A. Guérin and A. Orda, "QoS routing in networks with inaccurate information: theory and algorithms," IEEE/ACM Transactions on Networking, vol. 7, no. 3, pp. 350-364, 1999.

[2] D. H. Lorenz and A. Orda, "QoS routing in networks with uncertain parameters," IEEE/ACM Transactions on Networking, vol. 6, no. 6, pp. 768-778, 1998.

[3] L. Hanzo II and R. Tafazolli, "A survey of QoS routing solutions for mobile ad hoc networks" IEEE Communications Surveys \& Tutorials, vol. 9, no. 2, pp. 50-70, 2007.

[4] C. R. Lin and J.-S. Liu, "QoS routing in ad hoc wireless networks," IEEE Journal on Selected Areas in Communications, vol. 17, no. 8, pp. 1426-1438, 1999.

[5] L. Zhang, L. B. Cai, M. Li, and F. H. Wang, "A method for least-cost QoS multicast routing based on genetic simulated annealing algorithm," Computer Communications, vol. 32, no. 1, pp. 105-110, 2009.

[6] X. Yuan and X. Liu, "Heuristic algorithms for themulti-constrained quality of service routing," inProceedings of the 20th Annual Joint Conference of the IEEE Computer and Communications Societies (INFOCOM '01), vol. 2, pp. 844-853, IEEE, 2001.

[7] M. Abolhasan, T. Wysocki, and E. Dutkiewicz, "A review of routing protocols for mobile ad hoc networks," Ad Hoc Networks, vol. 2, no. 1, pp. 1-22, 2004.

[8] J. Xie, R. R. Talpade, A. Mcauley, and M. Liu, "AMRoute: ad hoc multicast routing protocol," Mobile Networks and Applications, vol. 7, no. 6, pp. 429-439, 2002.

[9] Y. S. Chen, Y. C. Tseng, J. P. Sheu, and P. H. Kuo, "An on-demand, link-state, multi-path QoS routing in a wireless mobile ad-hoc network," Computer Communications, vol. 27, no. 1, pp. 27-40, 2004.

[10] R. Vaishampayan and J. J. Garcia-Luna-Aceves, "Efficient and robust multicast routing in mobile ad hoc networks" in Proceedings of the IEEE International Conference on "Mobile Ad-hoc and Sensor Systems", pp. 304-313, IEEE, October 2004.

[11] P. Khadivi, S. Samavi, and T. D. Todd, "Multi-constraint QoS routing using a new single mixed metrics,"Journal of Network and Computer Applications, vol. 31, no. 4, pp. 656-676, 2008.

[12] S. S. Manvi and M. S. Kakkasageri, "Multicast routing in mobile ad hoc networks by using a multiagent system" Information Sciences, vol. 178, no. 6, pp. 1611-1628, 2008.

[13] F. Kuipers, P. Van Mieghem, T. Korkmaz, and M. Krunz, "An overview of constraint-based path selection algorithms for QoS routing,” IEEE Communications Magazine, vol. 40, no. 12, 2002. 
[14] K. Kunavut and T. Sanguankotchakorn, "Multi-Constrained Path (MCP) QoS routing in OLSR based on multiple additive QoS metrics," in Proceedings of the International Symposium on Communications and Information Technologies (ISCIT '10), pp. 226-231, IEEE, Tokyo, Japan, October 2010.

[15] L. Liu and G. Feng, "Simulated annealing based multi-constrained QoS routing in mobile ad hoc networks," Wireless Personal Communications, vol. 41, no. 3, pp. 393-405, 2007.

[16] S. Bitam and A. Mellouk, "Bee life-based multi-constraints multicast routing optimization for vehicular ad hoc networks," Journal of Network and Computer Applications, vol. 36, no. 3, pp. 981991, 2013.

[17] N. Ghaboosi and A. T. Haghighat, "Tabu search based algorithms for bandwidth-delay-constrained least-cost multicast routing," Telecommunication Systems, vol. 34, no. 3-4, pp. 147-166, 2007.

[18] Z. Subing and L. Zemin, "A QoS routing algorithm based on ant algorithm," in Proceedings of the IEEE International Conference on Communications (ICC '01), vol. 5, pp. 1581-1585, IEEE, 2001.

[19] C.-H. Chu, J. Gu, X. D. Hou, and Q. Gu, "A heuristic ant algorithm for solving QoS multicast routing problem," in Proceedings of the IEEE Congress on Evolutionary Computation (CEC '02), vol. 2, pp. 1630-1635, IEEE, Honolulu, Hawaii, USA, May 2002.

[20] R. Forsati, A. T. Haghighat, and M. Mahdavi, "Harmony search based algorithms for bandwidthdelay-constrained least-cost multicast routing" Computer Communications, vol. 31, no. 10, pp. 2505$2519,2008$.

[21] R. F. Abdel-Kader, "Hybrid discrete PSO with GA operators for efficient QoS-multicast routing," Ain Shams Engineering Journal, vol. 2, no. 1, pp. 21-31, 2011.

[22] P. Chen and T. L. Dong "A fuzzy genetic algorithm for QoS multicast routing" Computer Communications, vol. 26, no. 6, pp. 506-512, 2003.

[23] X. S. Yang and S. Deb, "Engineering optimization by cuckoo search," International Journal of Mathematical Modelling and Numerical Optimisation, vol. 1, no. 4, pp. 330-343, 2010.

[24] S. Walton, O. Hassan, K. Morgan, and M. R. Brown, "Modified cuckoo search: a new gradientfreeoptimization algorithm," Chaos, Solitons \& Fractals, vol. 44, no. 9, pp. 710-718, 2011.

[25] X. S. Yang and S. Deb, "Cuckoo search: recent advances and applications," Neural Computing and Applications, vol. 24, no. 1, pp. 169-174, 2014.

[26] O. Baskan, "Determining optimal link capacity expansions in road networks using cuckoo search algorithm with levy flights," Journal of Applied Mathematics, vol. 2013, Article ID 718015, 11 pages, 2013.

[27] P. Civicioglu and E. Besdok, "A conceptual comparison of the Cuckoo-search, particle swarm optimization, differential evolution and artificial bee colony algorithms" Artificial Intelligence Review, vol. 39, no. 4, pp. 315-346, 2013.

[28] P. I. Basarkod, S. S. Manvi, and D. S. Albur, "Mobility-based estimation of node stability in MANETs", in Proc. Int. Conf. Emerg. Trends in Comput., Commun. and Nanotechnol. ICE-CCN 2013, Tuticorin, India, 2013, pp. 126-130. 
[29] K. S. Trivedi, Probability and Statistics with Reliability, Queuing, and Computer Science Applications, 2 ed. Wiley Interscience, 2005

[30] P. I. Basarkod and S. S. Manvi, "Multiple parameters based approach to estimate bandwidth in mobile ad hoc networks", Int. J. Comp. Sci. Issues, Special Issue (IJCSI), vol. 1, no. 1, pp. 37-43, 2011.

[31] Woongsoo Na, Yunseong Lee, Jongha Yoon, Junho Park, and Sungrae Cho, "Fully Distributed Multicast Routing Protocol for IEEE 802.15.8 Peer-Aware Communication", International Journal of Distributed Sensor Networks Volume 2015.

\section{AUTHORS}

M. Vijayalakshmi Completed B.E and M.E in E.C.E from Osmania University. Presently pursuing Ph.D in the area of Wireless Networks at Jawaharlal Nehru Technological University, Hyderabad,(JNTUH) Under the guidance of Prof., Dr.D.Sreenivasarao, JNTUH. And working as an Assoc.Prof. in G.N.I.T.S., Hyderabad. Total teaching experience is of 16 years. 